\title{
RECIPROCAL VENTRICULAR EXTRASYSTOLES
}

BY

\section{SCHAMROTH AND D. ROSENZWEIG}

From the Baragwanath Hospital and the University of the Witwatersrand, Johannesburg, South Africa

An impulse originating in the A-V node or ventricles may be conducted retrogradely through the A-V node. The retrograde impulse may, under certain circumstances, return anterogradely through the $\mathrm{A}-\mathrm{V}$ node to the ventricles giving rise to a reciprocal or return extrasystole. One explanation for this re-entry phenomenon is based on the concept of a functional vertical division or longitudinal dissociation of the A-V node into two pathways (Schmitt and Erlanger, 1928)-(A) a nonrefractory pathway that conducts retrograde impulses from the lower centre, and (B) a refractory pathway that blocks retrograde impulses from the lower centre but can conduct impulses anterogradely, i.e. it has the property of unidirectional conduction.

The impulse from the lower centre is thus conducted retrogradely through the non-refractory pathway, A, and after reaching the upper regions of the node is conducted anterogradely through the second pathway, B. To enable the retrograde impulse to re-enter the second pathway, deep retrograde penetration of the A-V node to a critical level is necessary. The level at which re-entry can occur will be referred to as the re-entry level.

In the case of A-V nodal rhythm this mechanism will result in an inverted "nodal" $P$ wave "sandwiched" between two normal QRS complexes (L in Fig. 1). Should this mechanism occur with
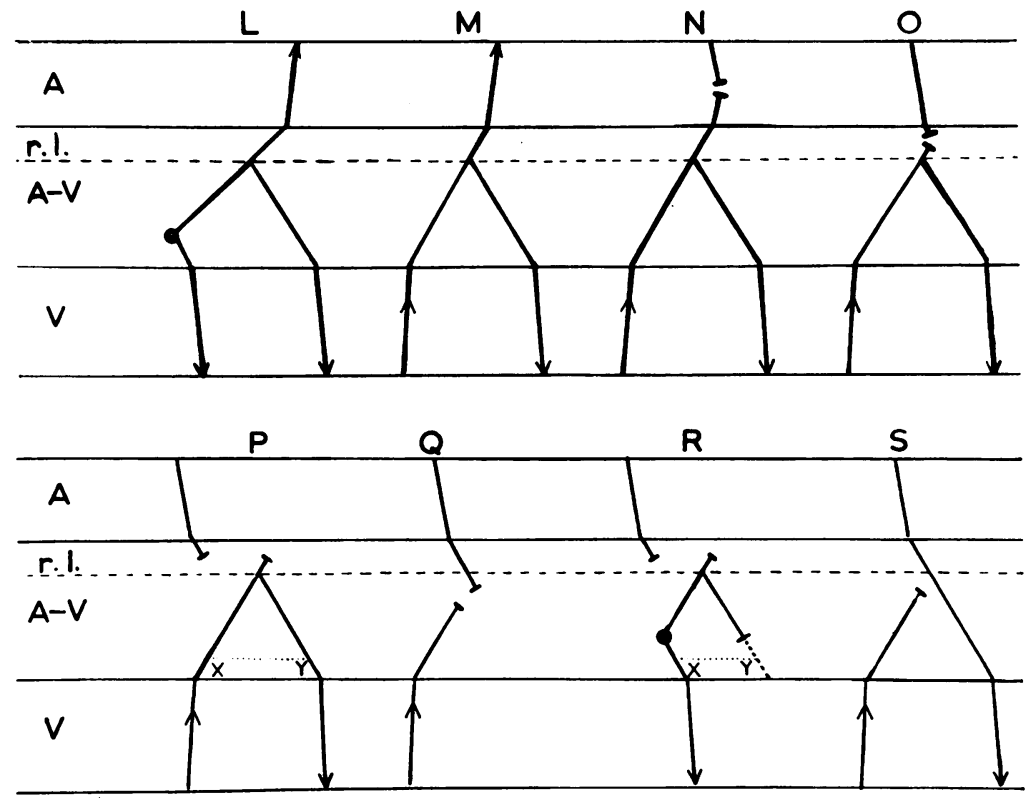

FIG. 1.-Diagrammatic illustration of various mechanisms in reciprocal rhythm. $\mathrm{A}=$ atrial level; $\mathrm{A}-\mathrm{V}=$ atrio-nodal level; $\mathrm{V}=$ =entricular level; the dotted line r.l. represents the re-entry level. Full explanation in text. 
a ventricular impulse, there will be a bizarre QRS complex followed by an inverted "retrograde" $P$ wave, which in turn is followed by a QRS complex resembling the normally conducted anterograde impulse ( $\mathrm{M}$ in Fig. 1).

We present a case where the timing of a non-conducted sinus impulse was such that it enabled the impulse from a ventricular extrasystole to penetrate deeply into the A-V node, reach the re-entry level, and return to the ventricles to record a reciprocal extrasystole. In addition, the sinus impulse rendered the upper part of the A-V node refractory thereby preventing atrial penetration by the ventricular impulse.

\section{Case Report}

The electrocardiograms were recorded during a period of digitalis intoxication in a 31-year-old African (negro) woman with hypertensive cardiac failure.

The tracing (Fig. 2, continuous strip of lead V1) shows a basic nodal rhythm with A-V dissociation; the $\mathbf{P}$ waves are not related to the normal nodal QRS complexes (labelled A in Fig. 2). Ventricular extrasystoles

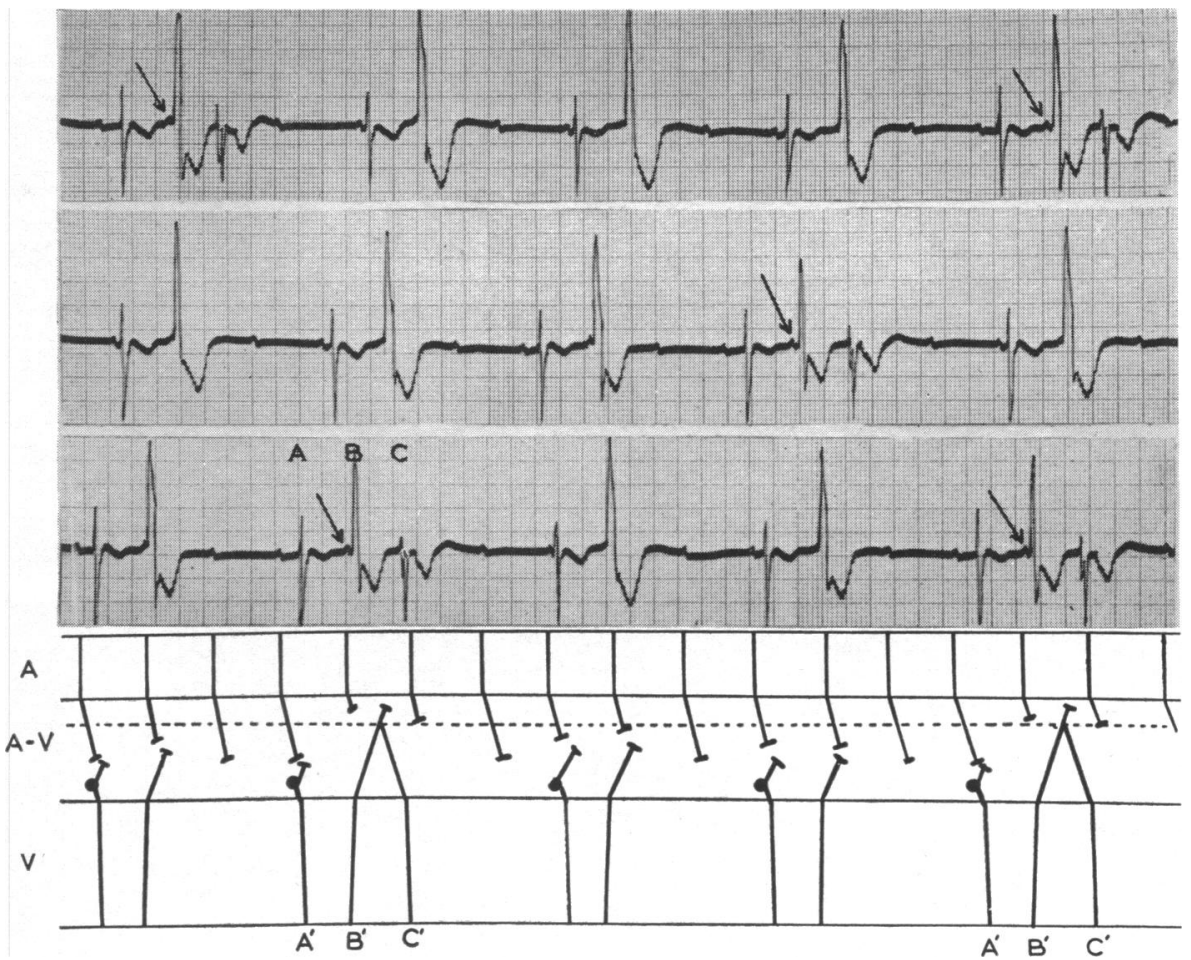

FIG. 2.-Electrocardiogram (continuous strip of lead V1). $\quad A=$ atrial level; $A-V=$ atrio-nodal level; $\mathrm{V}=$ =entricular level; the dotted line represents the re-entry level. Full explanation in text.

(labelled B in Fig. 2) are coupled to the nodal beats resulting in bigeminal rhythm. Complexes of different contour (labelled $\mathrm{C}$ in Fig. 2) are distributed throughout the tracing, which resemble complexes type A. Ventricular captures were not observed.

Distribution of the $C$ type complexes is critically related to the timing of the $P$ waves. Thus, complex type $C$ occurs whenever a $P$ wave falls just before complex type $B$ (arrows in Fig. 2). Complex type $C$ never occurred with the $\mathbf{P}$ waves in other positions. In addition, the $\mathrm{C}$ type complexes are constantly coupled to the B type complexes (coupling interval, $0.40 \mathrm{sec}$.). The following day the electrocardiogram showed an 
uncomplicated A-V nodal rhythm with A-V dissociation and neither the $B$ nor the $\mathrm{C}$ type complexes were present.

Thus the appearance of complex type $C$ is related to two factors: (1) the timing of the $P$ waves and, (2) the presence of the ventricular extrasystole, complex type B.

\section{Discussion}

The basic nodal rhythm occurs with A-V dissociation, and the absence of ventricular captures indicates that the sinus impulses do not penetrate beyond the A-V junction. The absence of inverted "retrograde" $P$ waves indicates that the nodal and ventricular impulses do not penetrate retrogradely beyond the $\mathrm{A}-\mathrm{V}$ junction. Interference between the sinus and the nodal or ventricular impulses thus takes place within the A-V node. Different degrees of anterograde and retrograde penetrations occur depending upon the varying time relations of the sinus and nodal or ventricular impulses (Fig. 2). Should the sinus impulse occur at a critical moment, viz. relatively early in relation to the ventricular impulse (arrows in Fig. 2), it will permit deeper retrograde penetration of the ventricular impulse (mechanism illustrated in P of Fig. 1). The ventricular impulse is thus able to reach the re-entry level (dotted lines in Fig. 1 and 2) and return anterogradely to record the reciprocal extrasystole - complex type $\mathbf{C}$ (Fig. 2; P in Fig. 1). The returning impulse finds the conducting system partially recovered and is therefore conducted with some degree of aberration. The sinus impulse also renders the upper part of the A-V node-the part above the re-entry levelrefractory: the retrograde impulse is thus unable to penetrate through into the atria. Should the timing of the impulse be such that it occurs at a position other than the critical moment described above, it will penetrate deeper into the A-V node and prevent the ventricular impulse from reaching the re-entry level (illustrated in $\mathrm{Q}$ of Fig. 1).

An alternative mechanism could be postulated for this arrhythmia, viz. that the related sinus impulses occur during the supernormal phase of conduction and are thus conducted to the ventricles to record the complex type $\mathrm{C}$ (mechanism illustrated in $\mathrm{S}$ of Fig. 1). However, this postulate is not tenable as the electrocardiogram recorded on the following day revealed that both the B and C type complexes were absent. Complexes type $\mathrm{C}$ were not recorded despite the occurrence of sinus impulses during all phases of the cycle.

It is noteworthy that although the sinus impulses will at times bear the same critical relation to the nodal as they do to the ventricular impulses, return extrasystoles do not follow nodal impulses. Nevertheless, return of the nodal impulse will occur under circumstances similar to that governing return of the ventricular impulse: however, the returning nodal impulse finds the region below the nodal pacemaker refractory due to preceding anterograde activation from the nodal impulse. Thus the interval between the initiating and returning nodal impulses in the region below the nodal pacemaker (illustrated as $\mathrm{X} \ldots \mathrm{Y}$ in $\mathrm{R}$ of Fig. 1) is short. This is in contrast to the longer interval between the initiating and returning ventricular impulses at the same level (illustrated as $\mathrm{X}$... Y in $\mathrm{P}$ of Fig. 1). The longer interval allows sufficient time for recovery of the lower junctional tissues, thereby permitting the returning impulse to penetrate through the ventricles.

Clinical observations of reciprocal ventricular extrasystoles are rare. Cases of reciprocal ventricular extrasystoles with retrograde activation of the atria were reported by Schott (1951), Kistin and Landowne (1951 - their Case 4), and by Scherf and Schott (1953 - their Fig. 84 and 85). Levin (1941) described a case of return extrasystoles from both nodal and ventricular impulses associated with retrograde activation of the atria.

Langendorf, Katz, and Simon (1944) have shown that the "sandwiching" of an inverted P wave between two QRS complexes is not an integral part of reciprocal rhythm. They describe a case where the type of atrial deflection is dependent upon the relative times of discharge of the sinus and ventricular impulses. Three types of $P$ waves were noted.

1. An inverted $P$ wave "sandwiched" between two QRS complexes when the timing was such that the retrograde impulse activated the atria completely (illustrated in $\mathbf{M}$ of Fig. 1). 
2. A fusion P wave "sandwiched" between two QRS complexes when both the impulses from the sinus and lower centres activated the atria (illustrated in N of Fig. 1).

3. A dissociated sinus P wave "sandwiched" between two QRS complexes when the impulses from the two centres interfered within the A-V node itself (illustrated in $\mathrm{O}$ of Fig. 1).

Bix and Marriott (1959) also describe a case of reciprocal beat, occurring during nodal rhythm, where a fusion $P$ wave was "sandwiched" between two QRS complexes.

Our case is unusual in that no P waves were "sandwiched" between the QRS complexes of the ventricular and reciprocal extrasystoles, and that re-entry was inferred from the time relationship of the sinus impulse to the ventricular impulse. It illustrates that for the diagnosis of reciprocal ventricular extrasystoles the following two criteria must be satisfied.

1. The reciprocal beat must be related, i.e. constantly coupled, to the ventricular extrasystole.

2. There must be evidence of retrograde conduction within the A-V node: such evidence maý be provided by either the timing of the $\mathrm{P}$ waves and/or the shape of an intervening $\mathrm{P}$ wave-a fusion or inverted $\mathrm{P}$ wave.

\section{Summary}

A case is described where the timing of a non-conducted sinus impulse was such that it enabled the impulse from a ventricular extrasystole to penetrate retrogradely into the A-V node, reach a re-entry level, and return anterogradely to the ventricles to record a reciprocal ventricular extrasystole.

The sinus impulse also rendered the upper part of the A-V node refractory thereby preventing atrial penetration by the ventricular impulse. case.

We would like to thank Dr. I. Frack, Superintendent of Baragwanath Hospital, for permission to publish this

\section{References}

Bix, H. H., and Marriott, H. J. L. (1959). Amer. J. Cardiol., 4, 128.

Kistin, A. D., and Landowne, M. (1951). Circulation, 3, 738.

Langendorf, R., Katz, L. N., and Simon, A. J. (1944). Brit. Heart J., 6, 13.

Levin, E. (1941). Rev. argent. Cardiol., 8, 197.

Scherf, D., and Schott, A. (1953). Extrasystoles and Allied Arrhythmias. Heinemann, London.

Schmitt, F. O., and Erlanger, J. (1928). Amer. J. Physiol., 87, 326.

Schott, A. (1951). Proc. roy. Soc. Med., 44, 151. 